\title{
3D Printing of Photocatalytic Filters Using a Biopolymer to Immobilize $\mathrm{TiO}_{2}$ Nanoparticles
}

\author{
A. Sangiorgi, ${ }^{1}$ Z. Gonzalez, ${ }^{2}$ A. Ferrandez-Montero, ${ }^{2}$ J. Yus, ${ }^{2}$ A. J. Sanchez-Herencia, ${ }^{2}$ \\ C. Galassi, ${ }^{,}$A. Sanson, ${ }^{1}$ and B. Ferrari $\oplus^{2, z}$ \\ ${ }^{1}$ CNR-ISTEC, Institute of Science and Technology for Ceramics, National Research Council, 48018 Faenza, Italy \\ ${ }^{2}$ Instituto de Ceramica y Vidrio, CSIC, 28049 Madrid, Spain
}

\begin{abstract}
Titanium oxide-based photocatalytic filters were produced by Fused Deposition Modelling (FDM) using biopolymers obtained from renewable biomass resources. The thermoplastic route allows shaping composites through the immobilization of photoactive $\mathrm{TiO}_{2}$ nanoparticles in an environmentally friendly bioplastic such as the polylactic acid (PLA). Composites with an inorganic charge of 30 $\mathrm{wt} \%$ of $\mathrm{TiO}_{2}$ nanoparticles (NPs) exhibit a $100 \%$ methyl orange (MO) degradation after $24 \mathrm{~h}$ of light exposition due to the extremely uniform dispersion of the nanophase within the polymer matrix in the FDM feedstock. Surface modification of $\mathrm{TiO}_{2} \mathrm{NPs}_{\text {allows the }}$ optimization of the colloidal dispersion and stabilization of the inorganic charge in a PLA solution and hence, the optimal distribution of nano-photoactive points in the $\mathrm{TiO}_{2} / \mathrm{PLA}$ filaments and scaffolds. The proposed new route of processing improves the dispersion of nano-charges comparing with the traditional thermo-pressing routes used for mixing thermoplastics based composites, avoiding the thermal degradation of the polymer and providing a customised product. In this manuscript the evolution of photodegradation with the increase of $\mathrm{TiO}_{2}$ content in the composite and the variation of the filter geometry was evaluated.

(c) The Author(s) 2019. Published by ECS. This is an open access article distributed under the terms of the Creative Commons Attribution 4.0 License (CC BY, http://creativecommons.org/licenses/by/4.0/), which permits unrestricted reuse of the work in any medium, provided the original work is properly cited. [DOI: $10.1149 / 2.0341905$ jes]

(cc) BY
\end{abstract}

Manuscript submitted November 16, 2018; revised manuscript received January 7, 2019. Published February 27, 2019. This paper is part of the JES Focus Issue on Semiconductor Electrochemistry and Photoelectrochemistry in Honor of Krishnan Rajeshwar.

Energy and environmental issues are among the most dramatic and urgent challenges that modern society has to face. Realizing that, researchers are spending lot of effort on more eco-friendly alternative technologies for many daily life areas. Among them, sustainable energy production and pollutant removal are two of the areas in which intense research is being carried out. Semiconductor-assisted photocatalysis (mainly based on Titanium dioxide, $\mathrm{TiO}_{2}$ ) is a well-established technique for water and air pollutants degradation. It is recognized as a predominant advanced oxidation process (AOP) thanks to its efficiency and eco-friendliness. ${ }^{1,2}$ In particular, AOP techniques of water purification mainly rely on the generation of $* \mathrm{OH}$ radicals and their homogeneous attack on organic molecules. About the latter, the spectrum of compounds that are susceptible to this approach includes dyes, pesticides, cosmetics, and more. $\mathrm{TiO}_{2}$, owing to its characteristics, is the most frequently used photocatalyst in water purification processes. It is relatively cheap, water insoluble, non-toxic and highly resistant to different chemicals. In the anatase form, it shows also the highest photocatalytic activity and resistance to so-called anodic photocorrosion. However, the application of $\mathrm{TiO}_{2}$ in form of suspended powder in contaminated water requires further additional operations for the separation after photocatalysis. To avoid this relevant technological inconvenience, several solutions are proposed, which enable the photocatalyst to be immobilised. $\mathrm{The} \mathrm{TiO}_{2}$ immobilization on various substrates is an interesting research area within the photocatalytic water treatment field. ${ }^{3}$ Furthermore, there are additional advantages achievable such as increasing the absorption properties, ${ }^{4-6}$ and surface hydroxyl groups or reducing the charge recombination. ${ }^{7}$

Immobilization of $\mathrm{TiO}_{2}$ can be done on powder and pellet substrates,${ }^{4-6}$ on rigid/thick substrates or on soft/thin materials. ${ }^{8-14}$ Some examples of powder/pellet substrates include activated carbon, ${ }^{5}$ magnesium-aluminum silicates ${ }^{6}$ and volcanic ash. ${ }^{4}$ The deposition of fine $\mathrm{TiO}_{2}$ powder on rigid glass surfaces is a quite accepted method. It is commonly accomplished by the simple coverage of the surface with water suspended titania powder and the relative drying. The asprepared films strongly adhere to the glass due to $\mathrm{TiO}_{2}$ electrostatic charge (the glass surface is usually negatively charged). $\mathrm{TiO}_{2}$ immobilized on soft substrates are normally referred as $\mathrm{TiO}_{2}$ films, membranes or filters. Some examples include $\mathrm{TiO}_{2}$ immobilized on porous

zE-mail: bferrari@icv.csic.es
$\mathrm{Al}_{2} \mathrm{O}_{3},{ }^{9-11}$ glass filter, ${ }^{13}$ polyvinylidene difluoride, ${ }^{12}$ sponge $^{8}$ and cellulose fibres. ${ }^{14}$ Various techniques, such as electrospinning, ${ }^{13,14}$ film casting, ${ }^{12}$ chemical vapour deposition, ${ }^{10,11}$ slip and dip-coating, ${ }^{9}$ have been investigated to achieve the $\mathrm{TiO}_{2}$ immobilization on soft substrates. Although many efforts have been spent to develop these functional filters, further research work is still needed to better understand both their operation and design. The latter is an important aspect in order to produce highly performing devices because it can produce, for e.g., fluid flux enhancements or fouling mitigation. The mentioned filter production techniques, such as slip or dip-coating, allow producing membranes with simple geometries, mainly with planar configuration. Thus, in order to overcome this limitation, we considered Additive Manufacturing (or three-dimensional printing) as a promising shaping technique.

Since the first technique for $3 \mathrm{D}$ printing became available in the late 1980s and was used to fabricate models and prototypes, ${ }^{15}$ AM technology has experienced more than 20 years of development and today is one of the rapidly developing advanced manufacturing techniques in the world due to its process flexibility, its ability to create complex structures and rapid response to design alterations. The 3D printing term indicates a class of technologies such as stereolithography (SLA), selective laser sintering (SLS), syringe extrusion, fused deposition modeling (FDM)/fused filament fabrication (FFF), etc. FDM is the one where a temperature controlled head extrudes a thermoplastic material (at temperatures above its glass transition temperature, $\mathrm{Tg}$ ) layer by layer. In this process, the material changes from a solid to a semi liquid state during the extrusion process and follows the path of a computer aided system. ${ }^{16}$ The common materials used in FDM process are acrylonitrile butadiene styrene (ABS), ${ }^{17}$ polycarbonate (PC), poly lactic acid (PLA), polyvinyl alcohol (PVA), etc. ${ }^{18}$ However, it is wellknown that one way to lend active chemistry to $3 \mathrm{D}$ printed thermoplastic objects is to incorporate inorganic nanoparticles into the polymer filaments. In this way, FDM technology was found to be a potential method for the fabrication of electronic sensors based on conductive carbon black filled poly (caprolactone) (PCL) filaments. ${ }^{16,19}$ Carbon nanotubes have been used to reinforce thermoplastic polyurethane filaments and to induce electrical conductivity. ${ }^{20-22}$ Finally, there has been interest to design and fabricate filaments and then devices, for space-based applications. ${ }^{16,23,24}$

Although recent works on $\mathrm{TiO}_{2}$-polymer nanocomposites, especially for biodegradable implants applications, ${ }^{25,26}$ can be found in 
literature, up to our knowledge there is no $3 \mathrm{D} \mathrm{TiO}_{2}$-based composites where the amount of the inorganic nanophase exceeds $5 \mathrm{wt} \%$. In this paper, we focus on the fabrication and characterization of composite filaments for FDM production of 3D photocatalytic filters. In this environmental friendly approach we selected PLA as polymer matrix, since it is a biopolymer obtained from renewable biomass resources, to have fashioning complex shaped photocatalytic filters. The $\mathrm{TiO}_{2}-\mathrm{PLA}$ filaments were initially prepared with $15-30 \mathrm{wt} \%$ of $\mathrm{TiO}_{2}$ nanoparticles (NPs) and tested in the photodegradation of methyl orange (MO) to demonstrate its photocatalytic activity.

\section{Materials and Methods}

All chemicals were of reagent grade and used without any further purification. The as-received $\mathrm{TiO}_{2}$ nanopowder (Aeroxide $\mathrm{P} 25$, Evonik Degussa $\mathrm{GmbH}$, Germany) with a density of $4.2 \mathrm{~g} / \mathrm{cm}^{3}$, a specific surface area of $50 \pm 15 \mathrm{~m}^{2} / \mathrm{g}$ and an anatase:rutile ratio of 80:20, was dispersed in deionized water to prepare a stable $\mathrm{TiO}_{2}$ water-based suspension formulated with a $6 \mathrm{vol} . \%$ of solid content by adding $6 \mathrm{wt} \%$ (referred to powder) of a stabilizer such as the branched polyethylenimine (PEI, pKa 8.6, Mw 25,000 mol/g, Aldrich, Germany) at $\mathrm{pH} 8$. Modified $\mathrm{TiO}_{2}$ NPs by PEI coverage in suspension were then ballmilled for 90 minutes (using $\mathrm{Al}_{2} \mathrm{O}_{3}$ balls of $1 \mathrm{~cm}$ in diameter) and subsequently centrifuged at $4000 \mathrm{rpm}$ for 15 minutes in order to remove the aqueous solvent.

The procedure used for the particles dispersion and the adsorption of PEI onto the NPs surfaces has been described previously elsewhere. ${ }^{27}$ However, to improve the centrifugation of well dispersed NPs, the conditions for the suspension coagulation were stabilised. In this sense, the modification of the zeta potential for $\mathrm{TiO}_{2} \mathrm{NPs}_{\text {induced }}$ by the absorption of PEI was determined in a wider $\mathrm{pH}$ range (2-12) using the laser Doppler velocimetry technique in a ZetasizerNano ZS (Malvern, UK), and then compared to zeta potential determination in a similar $\mathrm{pH}$ range for the bare nanopowder. The $\mathrm{pH}$ adjustment of the suspensions was carried out by addition of small quantities of $0.1 \mathrm{M}$ $\mathrm{HNO}_{3}$ or Tetramethylammonium Hydroxide (TMAH) and controlled with a $\mathrm{pH}$ probe (Metrohm AG, Germany). The particle size distribution of the PEI- $\mathrm{TiO}_{2}$ NPs was also characterized by Dynamic Light Scattering (DLS) by using the ZetasizerNano ZS (Malvern, UK) for concentration ranging between $0.001-0.1 \mathrm{~g} / \mathrm{L}$. All the mentioned suspensions were sonicated before each measurement using a UP400S Ultrasonication probe (Hielscher, Germany) for an optimized period of $30 \mathrm{~s}$.

Two different compositions of $\mathrm{PLA}^{\mathrm{TiO}}{ }_{2}(85: 15$ and 70:30) were prepared by mixing a specific amount of the modified PEI-TiO $\mathrm{NPs}_{2}$ (still wet after centrifugation) with a certain volume of a polylactic acid (PLA) solution, that was prepared at $80 \mathrm{~g} / \mathrm{L}$ dissolving, at $40^{\circ} \mathrm{C}$ under magnetic stirring, polymer pellets (PLA 2003D with D-isomer content of $4.25 \%$ provided by Natureworks (USA) in tetrahydrofuran (THF, Panreac Germany).

A vigorous mechanical stirring step coupled with a US treatment (30-60 seconds) were applied to obtain a homogeneous mix which was loaded in a conventional rotary evaporator to remove THF under reduced pressure. The resulting powder was completely dried in a laboratory stove at $80^{\circ} \mathrm{C}$ and milled later in a blade grinder to reduce the PLA:PEI-TiO ${ }_{2}$ granules size. The latter were extruded at temperatures ranging $185-187^{\circ} \mathrm{C}$ using a homemade single screw extruder to manufacture FDM filaments with a suitable diameter $(2.5-3 \mathrm{~mm})$. The morphology and shape of the modified PEI-TiO 2 NPs, the PLA:PEI$\mathrm{TiO}_{2}$ composite granules and, finally, the filaments were examined by a field emission scanning electron microscope (FESEM Hitachi S-4700, Japan).

The extruded filaments were employed for manufacturing scaffolds by FDM using a conventional 3D printer (Prusa I3 with the Repetier software) with a nozzle diameter ranging between $0.3-0.5 \mathrm{~mm}$. These filters were designed with a CAD software and printed varying parameters such as: filter diameter $(10,14$ and $2 \mathrm{~mm})$, scaffold height (number of printed layers: 4 or 8 ) and the infill (20-40\%). The bed and nozzle temperatures were fixed at 55 and $185^{\circ} \mathrm{C}$ respectively, with a filament feed rate of $40 \mathrm{~mm} / \mathrm{s}$. The printed filters were characterized by a Tabletop Scanning Electron Microscope (SEM, Hitachi TM-1000).

Thermogravimetric Analysis (TGA) (Perkin Elmer, USA) was used to study the thermal evolution of the PLA:PEI-TiO ${ }_{2}$ composites. Tests were performed in air atmosphere with a constant heating rate of $5^{\circ} \mathrm{C} / \mathrm{min}$ up to $600^{\circ} \mathrm{C}$. The identification of the crystal phases of the samples was carried out by X-ray diffraction (XRD) with a Siemens D5000 diffractometer (Germany) with a Kristalloflex 710 generator $(\mathrm{K} \alpha(\mathrm{Cu}) \lambda=1.5405 \AA ; 40 \mathrm{kV} ; 30 \mathrm{~mA} ; 2 \theta=5-70)$.

Finally, the photocatalytic activity of the scaffolds was evaluated by the degradation of methyl orange (MO) in aqueous solution. ${ }^{28}$ The used light source (Oriel, model 96000) is equipped with a solar light simulating Xe-arc lamp (Osram XBO $450 \mathrm{~W}$ ) with commercial AM 6197 filter. The tests were performed using $25 \mathrm{~mL}$ of $\mathrm{MO}$ aqueous solution with a concentration of $3 \mathrm{mg} / \mathrm{L}$. The $\mathrm{pH}$ of the solution was adjusted to 2 using $\mathrm{HCl}$. The quartz reactor vessel was plugged to prevent MO solution evaporation. First, photolysis and dark tests (adsorption) were performed to confirm that the MO degradation is only associated with the PLA:PEI-TiO ${ }_{2}$ scaffolds and not to light irradiation and/or adsorption. Photocatalytic measurements were performed introducing the PLA:PEI-TiO 2 scaffolds in the MO solution that was maintained under continuous stirring and irradiation. Degradation phenomena were monitored using a home-made system described previously elsewhere. ${ }^{28}$ A narrow bandpass filter centered at $500 \mathrm{~nm}$ and bandwidth of $10 \mathrm{~nm}$ (Thorlabs, FB-500-10 full width at half maximum) was placed in front of a biased Silicon Photodetector (Thorlabs DET100A). The filter limits allow the light transmission in a wavelength range that matches the absorption band of MO $(508 \mathrm{~nm})$. Therefore, the intensity of light at the detector allows measuring the degradation of MO. All the elements were installed on an optical table to ensure stability and also, optically isolated in the dark to avoid the presence of other light sources. The output signal from the detector (voltage) was sent to a Keithley 2010 multimeter where it was recorded every 10 min using a Visual Basic homemade code.

\section{Results and Discussion}

The stabilization of $\mathrm{TiO}_{2}$ NPs in deionized water was firstly examined through zeta potential measurements. Figure 1a shows the variation of the zeta potential as a function of $\mathrm{pH}$ for bare NPs and also for NPs covered with the polyelectrolyte PEI as dispersant additive $\left(\mathrm{PEI}-\mathrm{TiO}_{2}\right)$. Two zones of maximum stability were displayed in the curve corresponding to the bare $\mathrm{TiO}_{2}$, where the surface charge is enough to provide repulsive interactions among the particles, far from the isoelectric point (IEP) located at $\mathrm{pH} 7$. One zone was located at acid $\mathrm{pH}(2-4)$ with zeta potential values close to $+40 \mathrm{mV}$ and the other at basic $\mathrm{pH}(8-12)$ with values ranging from -30 to $-50 \mathrm{mV}$. Although the surface charge balance of NPs was slightly higher at other $\mathrm{pH}$ values, the working $\mathrm{pH}$ was fixed in at 8 for the PEI adsorption, since it is more efficient in these conditions. The PEI used is a branched chain with primary, binary and ternary amino groups that maintains highly protonated (charged) at $\mathrm{pH} 8$ favouring the additive adsorption on negative $\mathrm{TiO}_{2}$ surfaces. Consequently after PEI addition, the PEI$\mathrm{TiO}_{2}$ suspension (at $\mathrm{pH}$ 8) exhibits a zeta potential of $+66 \mathrm{mV}$, due to the adsorbed protonated PEI, and a shear-thinning behavior with a manageable viscosity of $70 \mathrm{mPas}$ (at shear rate of $100 \mathrm{~s}^{-1}$ ). ${ }^{27}$

Moreover, the DLS measurements were also performed to determine the particle size after the surface modification. Figure $1 \mathrm{~b}$ shows the particle size distribution of the PEI-TiO $\mathrm{NPs}_{2}$ where one narrow peak, centred on $120 \mathrm{~nm}\left(\mathrm{D}_{\mathrm{V} 50}\right)$ was observed as additionally confirmed by the cumulative volume \% curve. Despite the high dispersion obtained, the modified particles presented a slight trend to the agglomeration as confirmed by the BET diameter $\left(\mathrm{D}_{\mathrm{BET}}\right)$. This parameter is calculated from the powder morphological properties, specific surface area, SSA, and density, $\rho,\left(D_{\mathrm{BET}}=6 / \rho \cdot \mathrm{SSA}\right)$, and allows the evaluation of the effect of the polyelectrolyte on the particles dispersion. A value of $\mathrm{D}_{\mathrm{BET}}$ close to $30 \mathrm{~nm}$ was obtained, which means that the PEI-TiO ${ }_{2}$ NPs presented a relatively low agglomeration factor of $3\left(\mathrm{~F}_{\mathrm{ag}}=\mathrm{D}_{\mathrm{V} 50} / \mathrm{D}_{\mathrm{BET}}\right)$. Figure 1c shows the morphological 

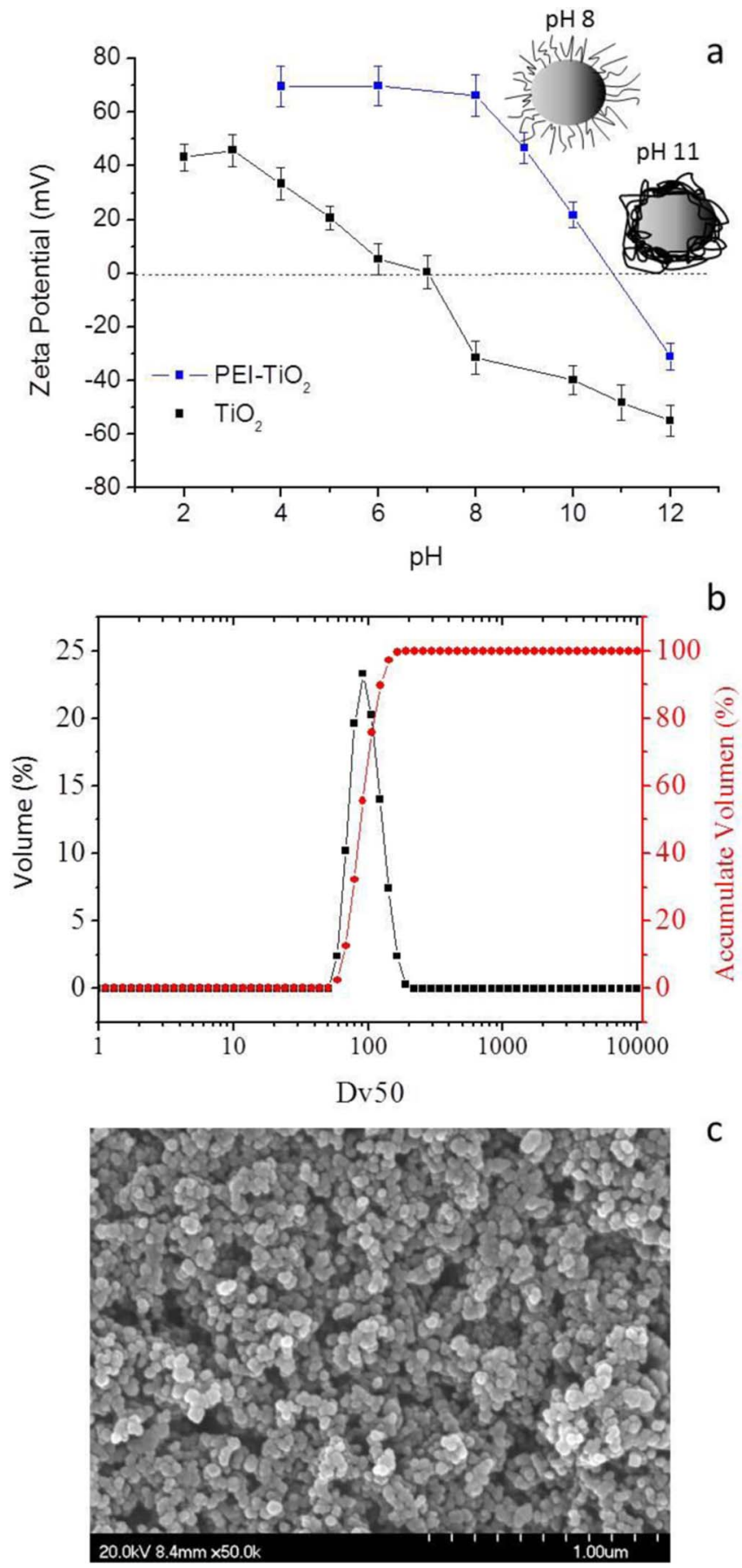

Figure 1. (a) Evolution of the zeta potential of bare- $\mathrm{TiO}_{2}$ and modified PEI$\mathrm{TiO}_{2} \mathrm{NPs}$ in aqueous suspension varying the $\mathrm{pH}(2<\mathrm{pH}<12)$; (b) Size distribution of the modified PEI-TiO 2 NPs and; (c) FESEM image of the modified $\mathrm{PEI}^{-\mathrm{TiO}_{2}}$ NPs.

characterization of the modified powder by FESEM that confirms the presence of small particles agglomerates around 100-150 nm. Although the size of the $\mathrm{TiO}_{2}$ agglomerates maintains in the nanometric range, the dispersion of $\mathrm{TiO}_{2}$ in the PLA matrix could be limited which can affect the photoactive efficiency of the 3D printed pieces.

The variation of the zeta potential as a function of $\mathrm{pH}$ was also examined after PEI adsorption at $\mathrm{pH}$ 8. The global charge balance at the particle surfaces was completely modified appearing positive up to $\mathrm{pH} 11$ after which the surface charge became negative. The variation of zeta potential induced by the $\mathrm{PEI}$ on the $\mathrm{TiO}_{2} \mathrm{NPs}$ can be explained by the way in which the polyelectrolyte chain interacts with the particle surface (through tail or train conformations), which depends on its pKa value, ionic strength, used solvent or molecular architecture. ${ }^{29}$ The amino groups of the PEI structure present a high degree of ionization when the working $\mathrm{pH}<\mathrm{pKa}$ (tail) and a lower degree when the working $\mathrm{pH}>\mathrm{pKa}$ (train). The sketches in Figure 1a illustrate both configurations. The zeta potential value of the PEI-TiO at $\mathrm{pH} 8$ was $+66 \mathrm{mV}$ while the increase of the $\mathrm{pH}$ toward higher basic values reduced the global charge, neutralizing the surface, to achieve the IEP of the system, where NPs are unstable and the system should coagulate.

In order to prepare the polymeric nanocomposite or feedstock for the extrusion of PLA/PEI-TiO ${ }_{2}$ filaments, ${ }^{30}$ the $\mathrm{TiO}_{2}$ NPs were firstly stabilized in aqueous suspensions by the addition of PEI, and then flocculated by varying the $\mathrm{pH}$ up to 11 to centrifuge them. The formation of $\mathrm{TiO}_{2}$ agglomerates $(100-150 \mathrm{~nm})$, as well as its low zeta potential at $\mathrm{pH} \mathrm{11}$, favours $\mathrm{TiO}_{2} \mathrm{NPs}$ flocculation and centrifugation. The wet flocculated PEI-TiO ${ }_{2}$ nanopowder was then mixed and re-dispersed in the PLA solution in THF under mechanical stirring. Figure 2a shows a scheme of the complete process. The as-prepared $\mathrm{PLA} / \mathrm{PEI}-\mathrm{TiO}_{2}$ suspension was then gently dried under reduced pressure obtaining PLA/PEI-TiO ${ }_{2}$ composite granules which were grinded in a blade grinder to reduce their size and to adapt them to the extruder conditions. These granules were then extruded in a single screw extruder with a thermal pass of only $10 \mathrm{~cm}$ at temperatures ranging from $185-187^{\circ} \mathrm{C}$, obtaining composite filaments with homogeneous diameters lower than $1.75 \mathrm{~mm}$, which were used as the PLA/PEI-TiO composite feedstock for printing. Figure $2 \mathrm{~b}$ displays the pictures of the obtained composites, both powder and filaments, for a specific composition of 85:15 (wt/wt) PLA:PEI-TiO

In deep microstructural analysis of the granules, filaments and scaffolds were also characterised by FESEM. Micrographs in Figures $3 \mathrm{a}$ and $3 \mathrm{~b}$ show a low magnification image of a PLA/PEI-TiO ${ }_{2}$ granule, where the inorganic nanoparticles (white arrows) are just slightly agglomerated but homogeneously distributed in the blend surrounding PLA (blue arrows). This well dispersed composite makes PLA/PEI$\mathrm{TiO}_{2}$ granules suitable candidates for the production of FDM filaments with an isotropic composition. Figure $3 \mathrm{c}$ shows an image of the cross section of an extruded filament $\left(185-187^{\circ} \mathrm{C}\right)$ which fulfils the requirements for printing. Although the appearance of the fracture surface is mostly plastic due to the high percentage of the polymer $(85: 15(\mathrm{wt} / \mathrm{wt})$ PLA:PEI-TiO 2 ), NPs agglomerates of $100-150 \mathrm{~nm}$, still visible at such magnification, appear to be well-distributed inside the filament (white arrows). The PEI-TiO ${ }_{2}$ NPs distribution in the PLA matrix is retained after printing at $185^{\circ} \mathrm{C}$. The micrographs of the PLA/PEI$\mathrm{TiO}_{2}$ scaffold in Figure 3d display the dense structure of the rods with inorganic NPs distributed both at the rod surfaces and inside parts

Figure 4a shows the XRD spectra of the 85:15 (wt/wt) PLA:PEI$\mathrm{TiO}_{2}$ granules and scaffolds. The pattern of the granules is characteristic of a mixture of $\mathrm{TiO}_{2}$ and a crystalline polymer, where the diffractions at $20.7^{\circ}$ and $22.9^{\circ}$ indicate the presence of the $\alpha$-phase of PLA (Figure $4 \mathrm{~b}$ ). ${ }^{31}$ The diffractogram of the scaffold shows the presence of amorphous PLA (from the wide band at $20^{\circ}$ ) derived by the thermal treatments during the extrusion and printing processes. On the other hand, the $\mathrm{TiO}_{2}$ phase maintains in both cases the expected ratio of anatase and rutile $95 \%$ and 5\%, compatible with the P25 nanopowder composition. These results evidence the effect of $\mathrm{TiO}_{2} \mathrm{NPs}$ as nucleant agent for the polymer matrix. When the composites are processed from the stable suspension of $\mathrm{PEI}_{-\mathrm{TiO}} \mathrm{NPs}_{2}$ in a PLA solution in fact, the excellent dispersion of PEI modified $\mathrm{TiO}_{2}$ NPs induces the precipitation of pure crystalline PLA over the inorganic seeds during drying. ${ }^{32}$ However, for the extrusion process, the $\mathrm{PLA}-\mathrm{TiO}_{2}$ granules are melted and then deposited: the XRD analysis clearly shows that the polymer preserved the amorphous state after solidification. PLA is in fact a biopolymer that does not crystallize from melting and therefore the thermal processing erases the matrix crystallinity.

Two different PLA/PEI-TiO 2 composites were used to prepare the feedstock granule-like: 85:15 and 70:30 (wt/wt PLA:PEI-TiO ${ }_{2}$ ), labelled respectively $15 \mathrm{TiO}_{2}$ and $30 \mathrm{TiO}_{2}$. The same printing parameters 


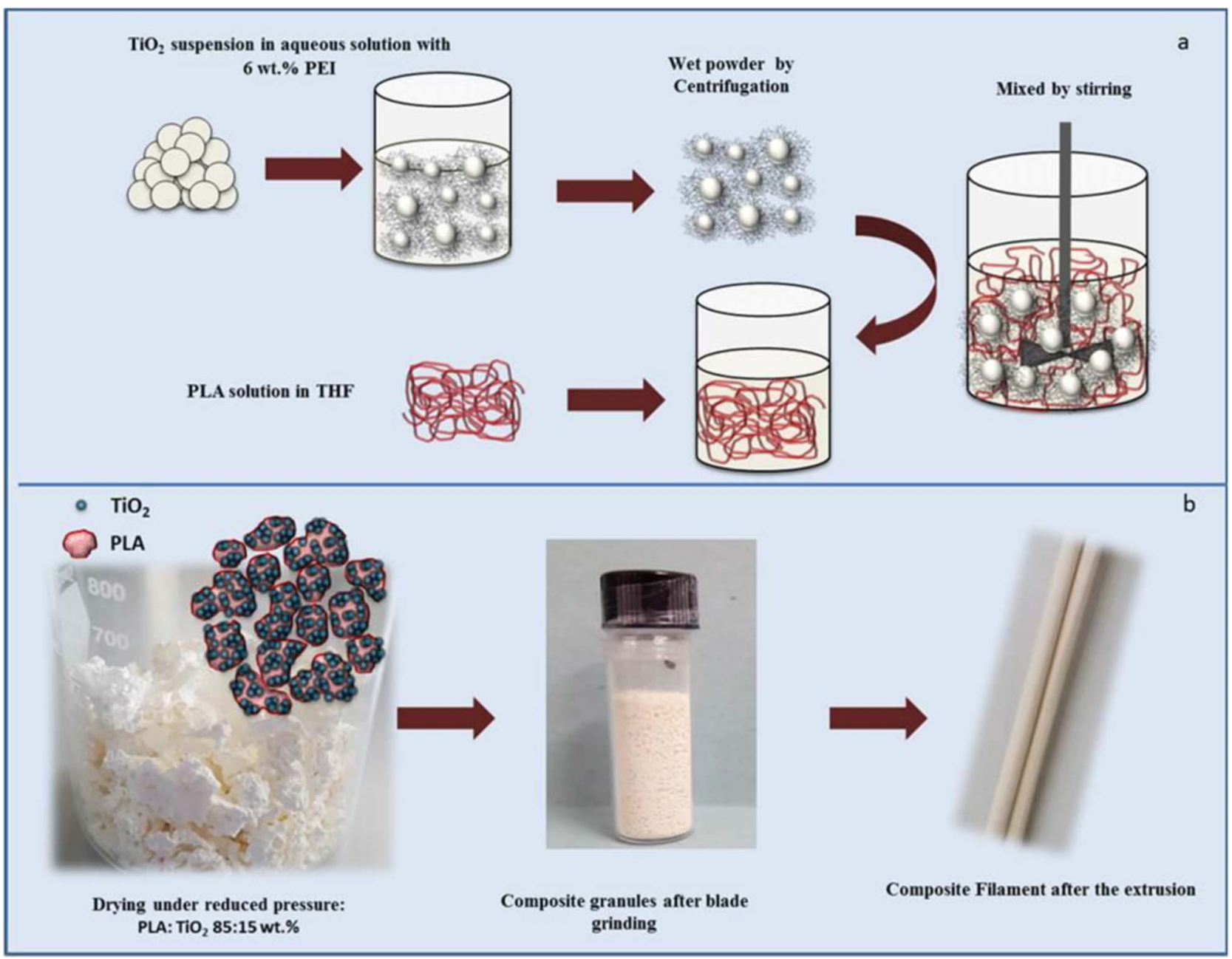

Figure 2. (a) Scheme of the process for the preparation of modified $\mathrm{PEI}-\mathrm{TiO}_{2}$ suspension in PLA and; (b) Pictures of the resulting dried composite, $\mathrm{PLA} / \mathrm{PEI}-\mathrm{TiO}{ }_{2}$ granules, after and before blade grinding as well as the composite filament after the extrusion.
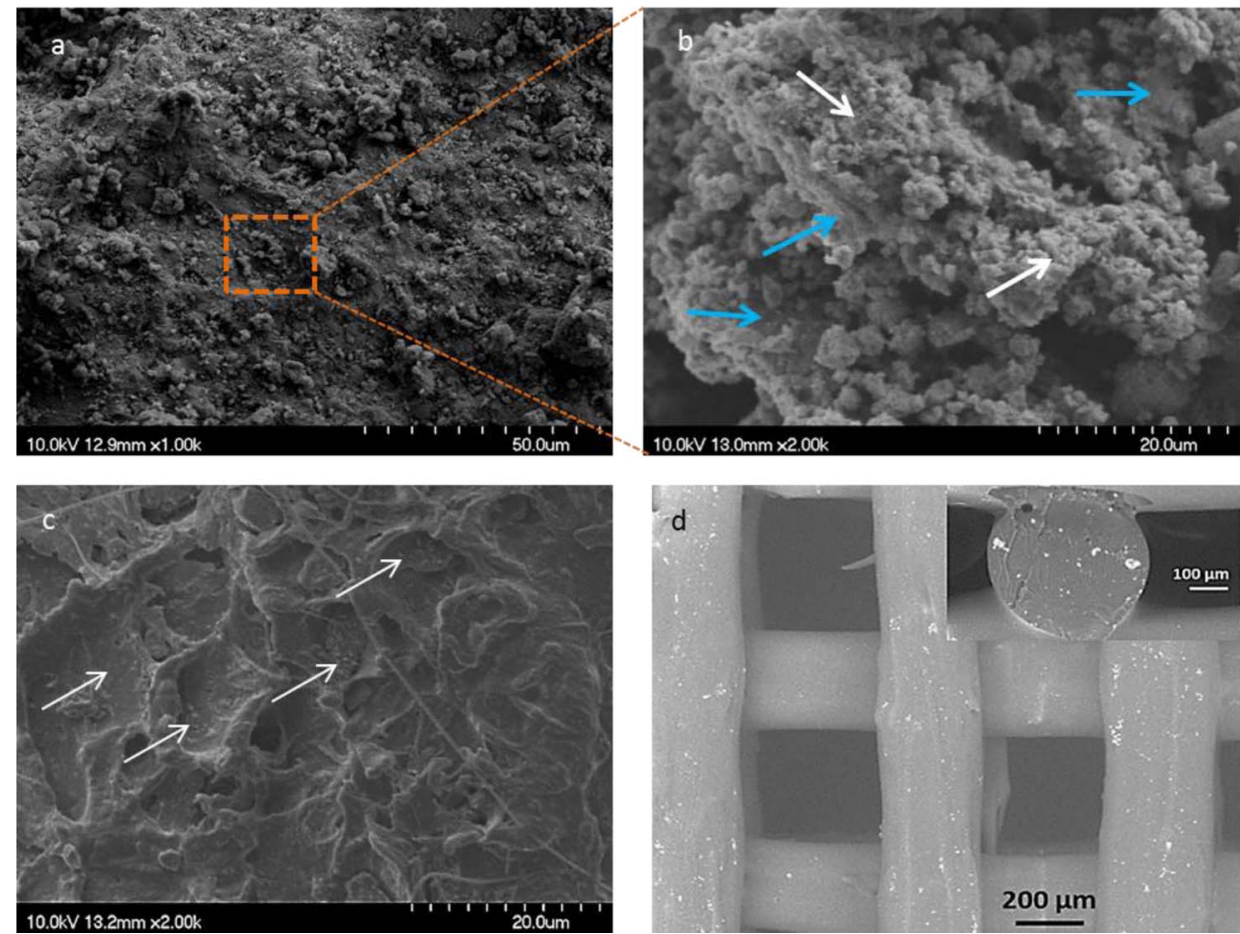

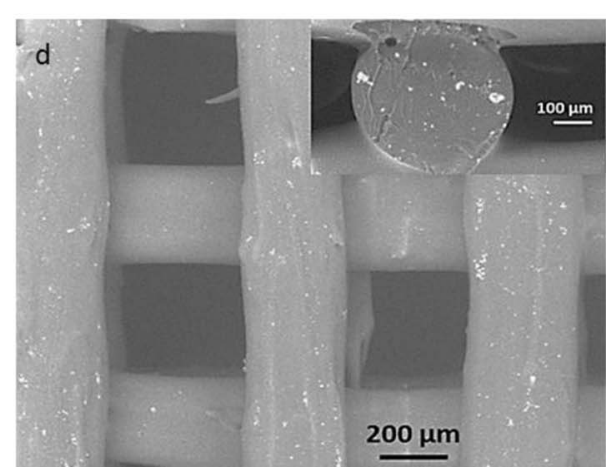

Figure 3. (a, b) FESEM images of the $\mathrm{PLA} / \mathrm{PEI}-\mathrm{TiO}_{2}$ granules at different magnifications, where blue and white arrows point to the PLA and $\mathrm{TiO}_{2}$ aggregates, respectively; (c) FESEM image of the cross section of a PLA/PEI$\mathrm{TiO}_{2}$ filament and; (d) SEM image of the top view of a filter including (inset) the detail of the rod cross-section. All images correspond to the composite 85:15 (wt/wt) PLA:PEI-TiO 2 . 


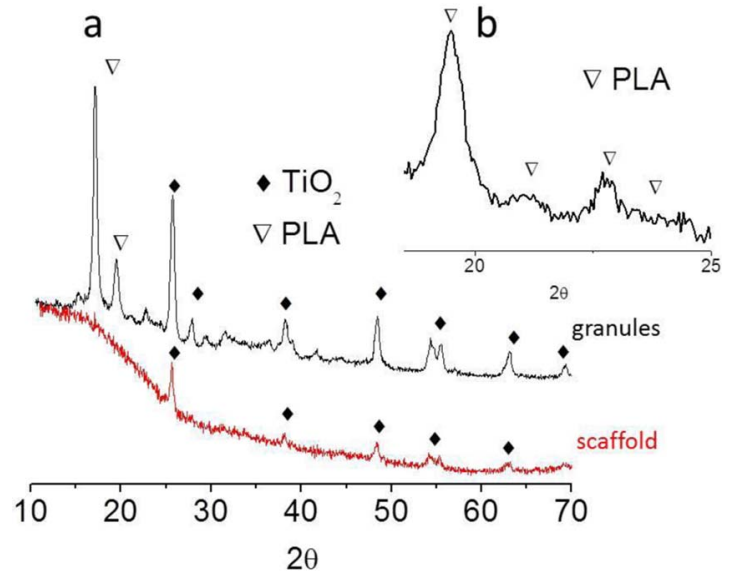

Figure 4. (a) XRD spectra of the granules and scaffold of the composite 85:15 (wt/wt) PLA:PEI-TiO ${ }_{2}$ and; (b) detail of the peaks associated with the $\alpha$ crystalline phase in the granules scanning.

(a printing nozzle of $0.5 \mathrm{~mm}$ in diameter, a filament feed rate of 40 $\mathrm{mm} / \mathrm{s}$ and, a bed and nozzle temperatures of 55 and $185^{\circ} \mathrm{C}$ respectively), were used to produce different filters designs: in particular filters with diameters of 10,14 and $20 \mathrm{~mm}$, heights of 4 or 8 layers and, infills ranging 20-40\% (which defines the free space left on the designed piece). Table I summarizes the most relevant printing conditions while Figure 5 shows the CAD printing shapes corresponding to the explored printing parameters: scaffold diameter, number of layers and infill. Table I also includes the calculated dimensions of the scaffold: height and the height $x$ length of the structure holes, (considering the CAD models which define the number of bars and holes of the designed scaffold structure, the nozzle diameter $(0.4 \mathrm{~mm})$ which define the diameter of the printed bars), the diameter of the filter (10, 14 and $20 \mathrm{~mm}$ ) and the number of layers (4 and 8 layers).

Figure $6 \mathrm{a}$ quantifies the MO photodegradation capability respectively of $15 \mathrm{TiO}_{2}$ and $30 \mathrm{TiO}_{2}$ filters, $\mathrm{F} 1$ and $\mathrm{F} 5$ in Table I, while their microstructure is reported in Figures $6 \mathrm{c}-6 \mathrm{~h}$. It is important to consider that both filters were printed following the same CAD model (F1 and F5 in Figure 5) having 8 layers and $10 \mathrm{~mm}$ of diameter. The preliminary photolysis and dark tests showed that neither photolysis nor adsorption processes occur. Plots in Figure 6a show that the designed porosity is higher enough to be infiltrated by the MO solution, and more than $50 \%$ MO degradation is recorded after $24 \mathrm{~h}$ with both compositions.

According to a great number of researchers, the rates of photocatalytic oxidation of various dyes, $\mathrm{MO}$ included, over illuminated $\mathrm{TiO}_{2}$ fitted the Langmuir-Hinshelwood kinetics model: ${ }^{33}$

$$
r=\frac{d C}{d t}=\frac{k K C}{1+K C}
$$

where $\mathrm{r}$ is the oxidation rate of the reactant, $\mathrm{C}$ is the concentration of the reactant, $\mathrm{t}$ the illumination time, $\mathrm{k}$ the reaction rate constant, and $\mathrm{K}$ is the adsorption coefficient of the reactant. When the initial chemical concentration of the dye $\left(\mathrm{C}_{\mathrm{o}}\right)$ is low, the above equation can be simplified to an apparent first-order equation:

$$
\ln \left(\frac{C o}{C}\right)=k t
$$

The plots of $\ln \left(\mathrm{C}_{\mathrm{o}} / \mathrm{C}\right)$ versus time $(\mathrm{t})$ represent a straight line with a slope $\mathrm{k}$ that is the apparent first-order rate reaction constant. ${ }^{34}$ These considerations are valid for $\mathrm{TiO}_{2}$ as powder samples or immobilized in a film or coating form. In this stage of the study, we assume to be in the same conditions to evaluate the MO degradation kinetic in presence of the hybrid filters. The obtained plots (Figure 6b) confirm that the MO degradation follows a first-order kinetic model, although it is important to note that the $30 \mathrm{TiO}_{2}$ filters achieve this degradation regimen after $5 \mathrm{~h}$. Moreover, $\mathrm{k}$ values demonstrate that the $\mathrm{F} 1\left(15 \mathrm{TiO}_{2}\right)$ filter exhibits better photocatalytic behavior in degrading MO. The differences in the reaction rates, or in the photocatalytic behavior, are usually associated with changes of the structure, texture and porosity of the microstructure. ${ }^{28}$

Comparing the two filters, the $\mathrm{F} 5\left(30 \mathrm{TiO}_{2}\right)$ shows less effectiveness in degrading the MO solution. Figures $6 \mathrm{c}-6 \mathrm{~h}$ show the SEM images of the top view and the cross section of both filters F1 (c, e and g) and F5 $(d, f$ and $h$ ) at different magnifications. Figures $6 \mathrm{c}$ and $6 \mathrm{~d}$ illustrate the $3 \mathrm{D}$ periodic structures composed, especially in the $\mathrm{F} 1$ case, by smooth surfaces and quite uniform cylindrical rods. However, observing the cross section of the scaffolds (Figures 6e-6h) the incorporation of a double amount of $\mathrm{TiO}_{2} \mathrm{NPs}$ promotes a partial collapse of the porous structure. Although the bed and nozzle temperature are kept constant for both composites, a regular printing is impeded by the presence of a higher inorganic charge amount finely dispersed within the PLA matrix. Irregular printing leads to the deposition of asymmetrical rods reducing and sometimes closing the pre-designed porosity of the filter, and then, lowering the filter surface area exposed to the dye molecules.

F1 and F5 filters dimensions are far from that designed (see Figure 5 and Table I). The whole scaffold height decreases down to $2.75 \mathrm{~mm}$, instead of the designed $3.20 \mathrm{~mm}$, since the holes height decreases down to $0.32 \mathrm{~mm}$ (versus $0.4 \mathrm{~mm}$ at the CAD model) due to the deformation of the bars during the layer-by-layer printing, evidenced in Figure 6e. Similarly the bars also deform acquiring lengths up to $0.59 \mathrm{~mm}$ (versus $0.4 \mathrm{~mm}$ at the CAD model), while the holes length decreases, ranging $0.43-0.56 \mathrm{~mm}$. Consequently the porosity decreases in $\mathrm{F} 1$ $\left(15 \mathrm{TiO}_{2}\right)$ filter if it is compared with the reference structure in Figure 5. This effect is more pronounced in the F5 filter $\left(30 \mathrm{TiO}_{2}\right.$ in Figure 6f). In fact the F5 scaffold height reduces to $2.00 \mathrm{~mm}$. At higher magnifications, the reduction of the spaces between bars in different layers, as well as the decreasing (even inexistent) spaces between bars in the same layer, are evident. Comparing the structures in Figures $6 \mathrm{~g}$ and $6 \mathrm{~h}$ also demonstrates that an increase of the NPs amount leads more defects inside the rods core (closed porosity) while the increase on NPs doesn't take place necessarily in the bars surface, and can reduce the photocatalytic effectivity. In view of the FESEM results,

\begin{tabular}{|c|c|c|c|c|c|c|c|}
\hline Filter & Label & Diameter $(\mathrm{mm})$ & Number of layers & Infill (\%) & Height* $^{*}(\mathrm{~mm})$ & Hole dimensions $*(\mathrm{~mm})$ : height $\times$ length & Weight $(\mathrm{g})$ \\
\hline F1 & \multirow{2}{*}{$15 \mathrm{TiO}_{2}$} & 10 & 8 & 40 & 3.2 & $0.4 \times 0.80$ & 0.12 \\
\hline F3 & & 14 & 4 & 25 & 1.2 & $0.3 \times 0.75$ & 0.11 \\
\hline F5 & \multirow{3}{*}{$30 \mathrm{TiO}_{2}$} & 10 & 8 & 40 & 3.2 & $0.4 \times 0.80$ & 0.12 \\
\hline F6 & & 14 & 4 & 40 & 1.6 & $0.4 \times 0.73$ & 0.14 \\
\hline F7 & & 20 & 4 & 40 & 1.6 & $0.4 \times 0.82$ & 0.18 \\
\hline
\end{tabular}

Table I. Summary of the PLA/PEI-TiO 2 filters characteristics processed by FDM. 

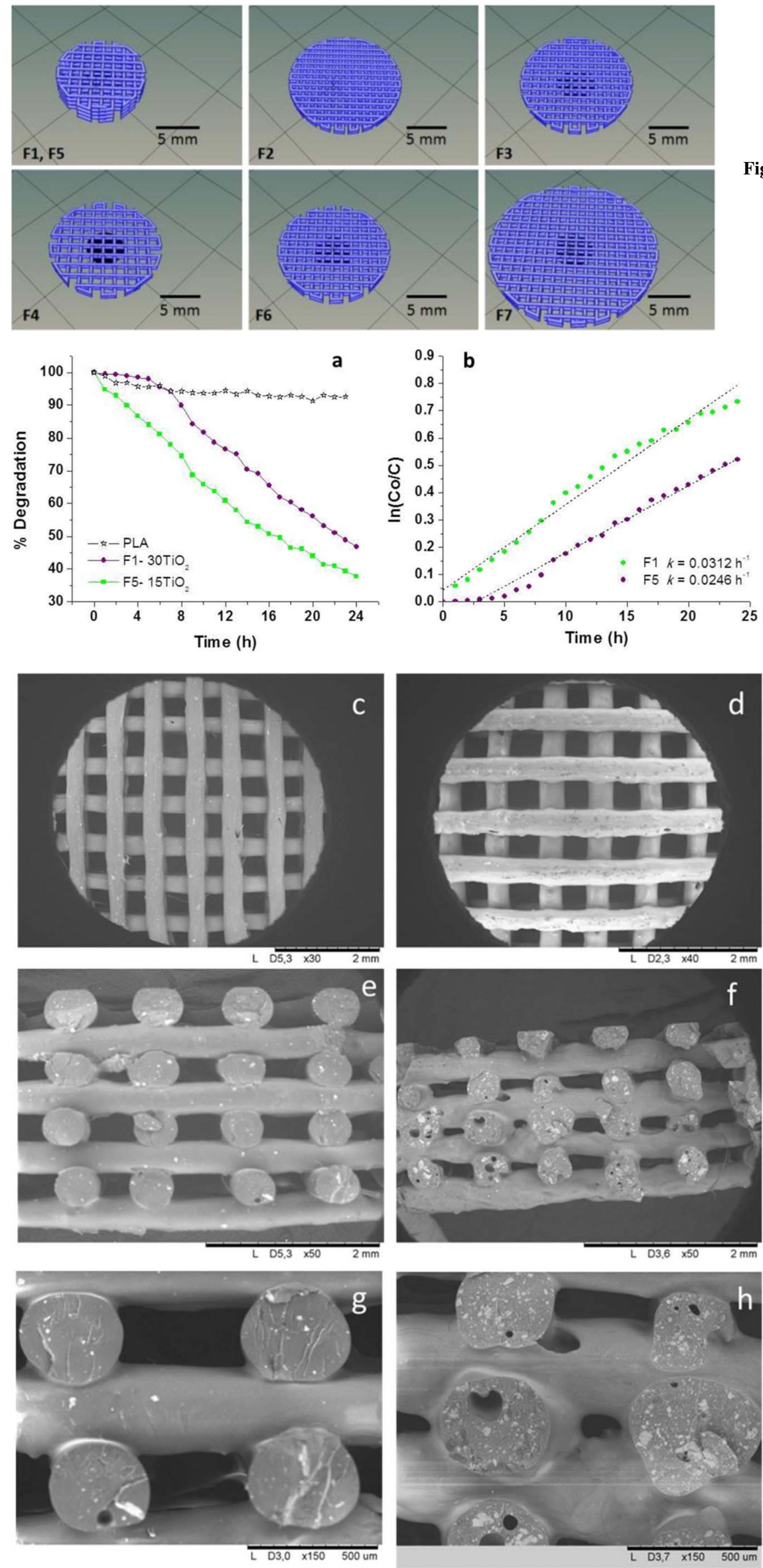

Figure 5. CAD models of the filters in Table I.

Figure 6. (a, b) Photodegradation of $\mathrm{MO}$ and kinetics of F1 and F5 filters; (c, e, g) FESEM images of the F1 filter; $(d, f, h)$ FESEM images of the F5 filter at different magnifications. 

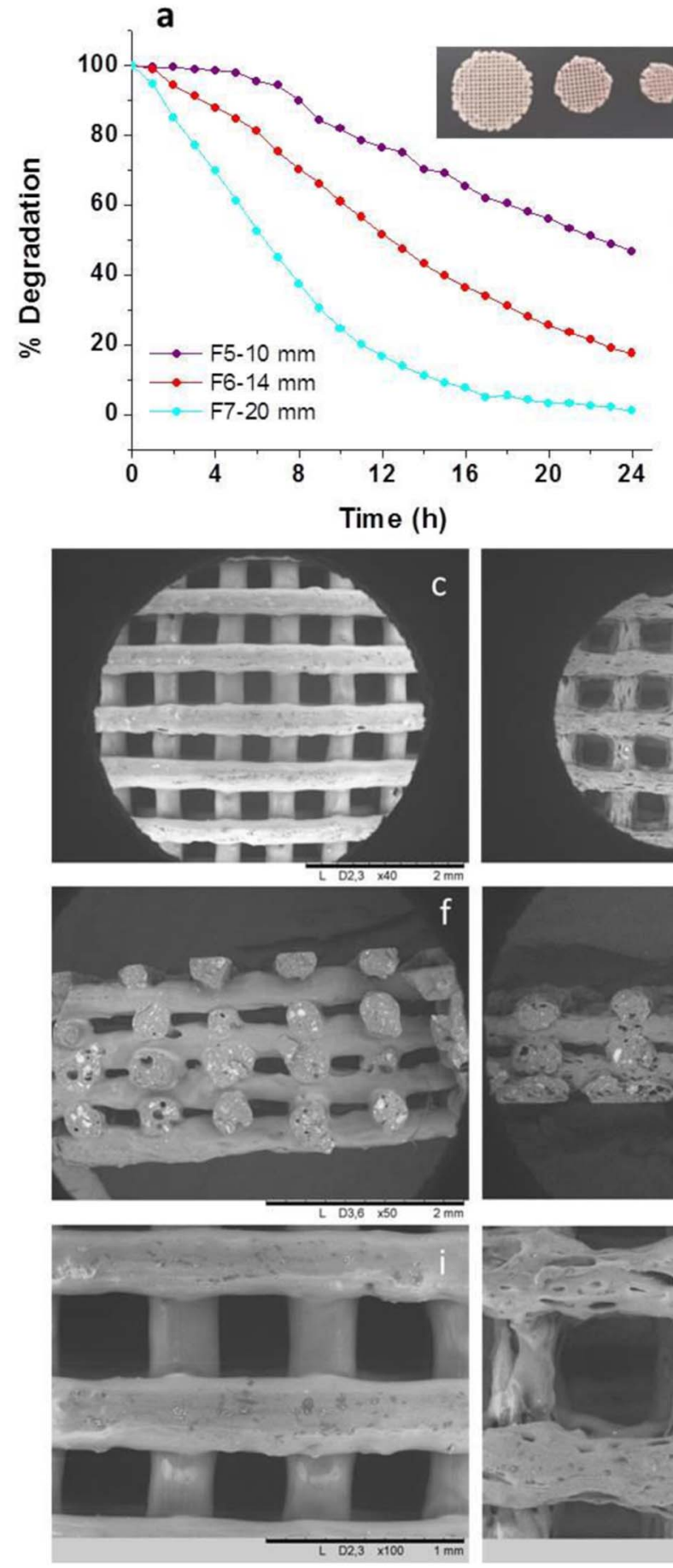

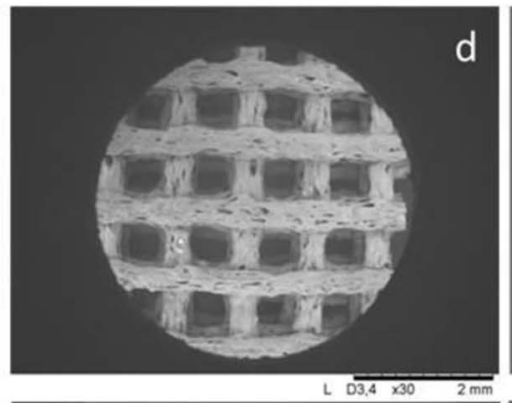

d
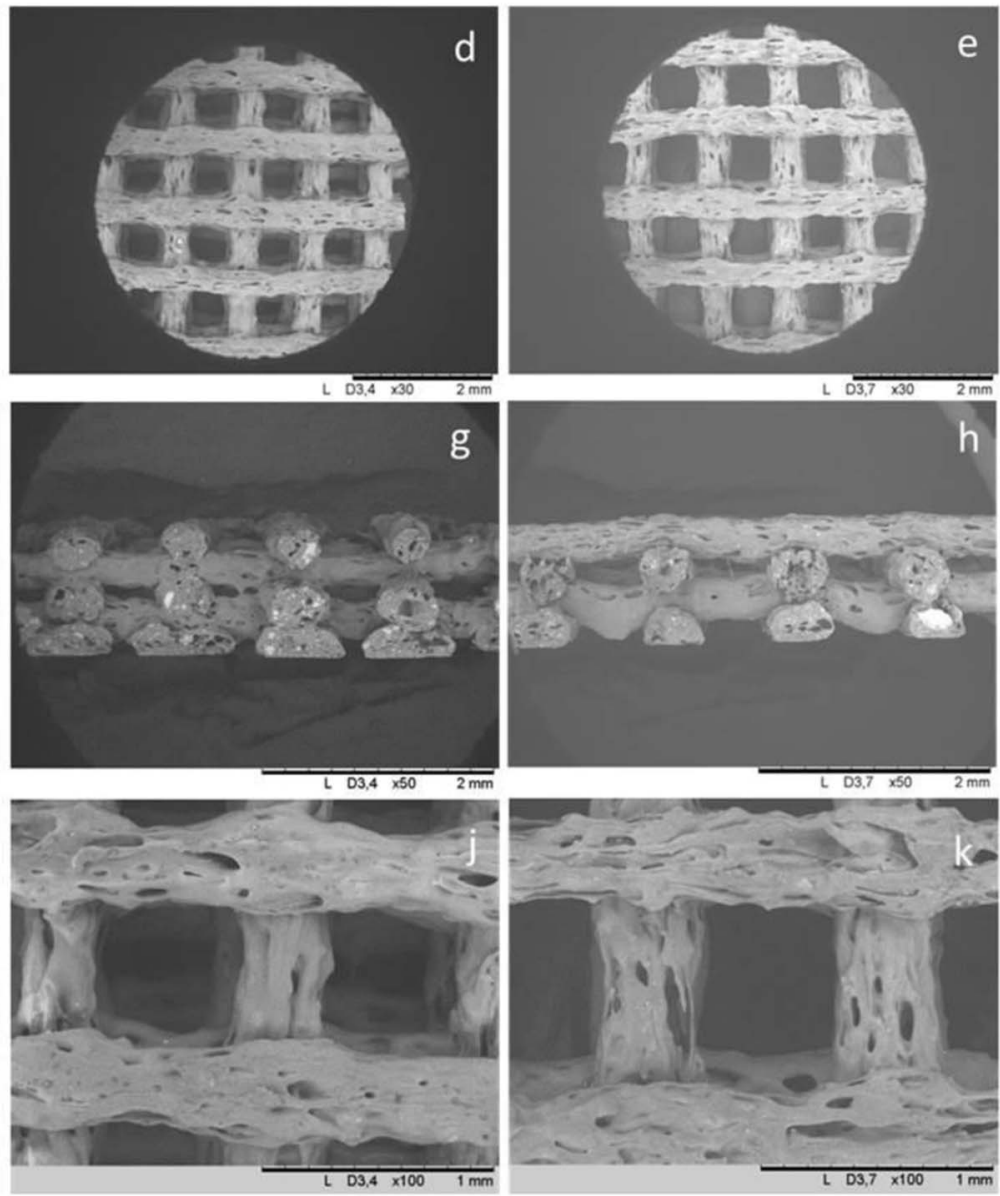

Figure 7. (a, b) Photodegradation of MO and kinetics of F5, F6 and F7 filters; (c, f, i) FESEM images of the F5 filter; (d, g, j) FESEM images of the F6 filter; (e, h, k) FESEM images of the F7 filter at different magnifications.

degradation kinetics could be affected by the fact that the NPs imbibed in the composite are partially available at the surface of the filter rods, but also the structure collapse impedes the homogeneous irradiation of all the structure due to partial shading effects between each other bars.

Figures $7 \mathrm{a}$ and $7 \mathrm{~b}$ quantified the $\mathrm{MO}$ photodegradation of $30 \mathrm{TiO}_{2}$ filters designed with $40 \%$ infill using nozzles of $0.4 \mathrm{~mm}$ of diameter, scaffold diameters of 10,14 and $20 \mathrm{~mm}$, and 8 or 4 layers, exhibiting weights ranging $0.12-0.18 \mathrm{~g}$, corresponding to the filter F5, F6 and F7 in Table I, which SEM images are showed in Figures $7 \mathrm{c}-7 \mathrm{~h}$ and insets, respectively.
The F7 filter (20 $\mathrm{mm}$ in diameter and 4 layers) shows the highest efficiency in degrading the MO solution, after 20 hours, a total degradation of the dye molecules is achieved. This effect is ascribed to the potential higher exposed surface area and the higher amount of $\mathrm{TiO}_{2}$ NPs included in the three-dimensional structure. Although scaffold bars maintain their diameter, the holes length differs from the CAD model (Figure 5 and Table I) similarly to what occurs in the F5 filter, that corresponds to the same feedstock composition $\left(30 \mathrm{TiO}_{2}\right)$. However, the wall-roughness of the rods plays an important role on the filters behavior. Comparing the Figures $7 \mathrm{i}, 7 \mathrm{j}$ and $7 \mathrm{k}$, extremely rough surfaces are illustrated going from the F5 $(10 \mathrm{~mm}$ in diameter and 8 

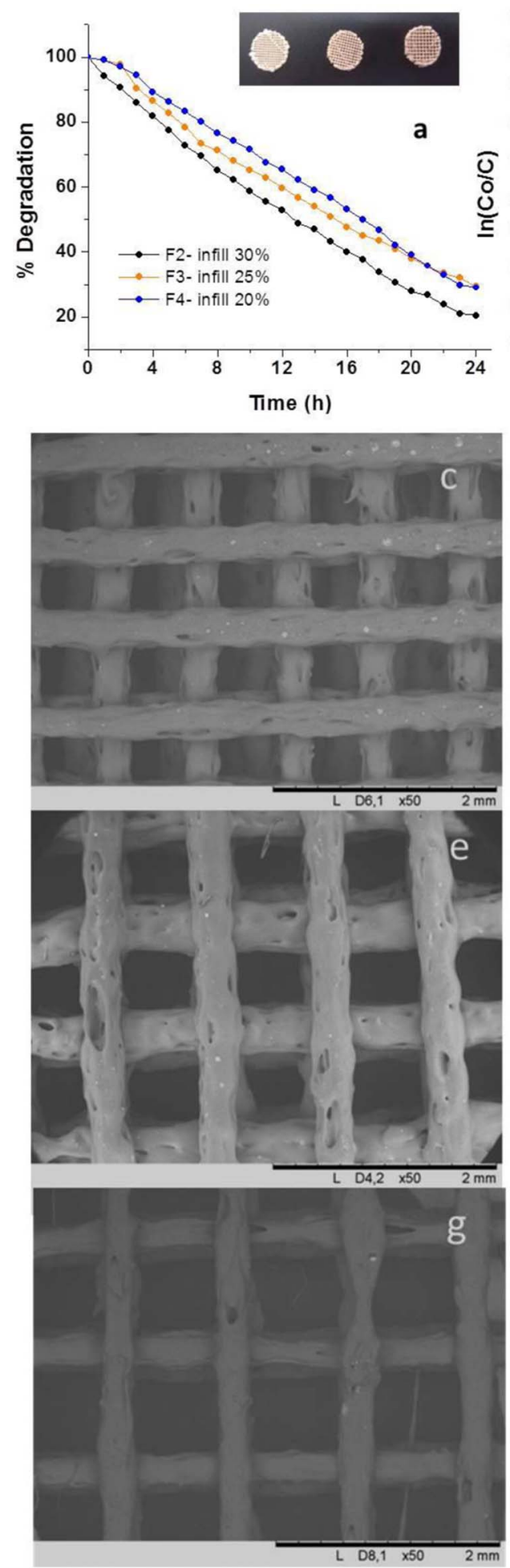
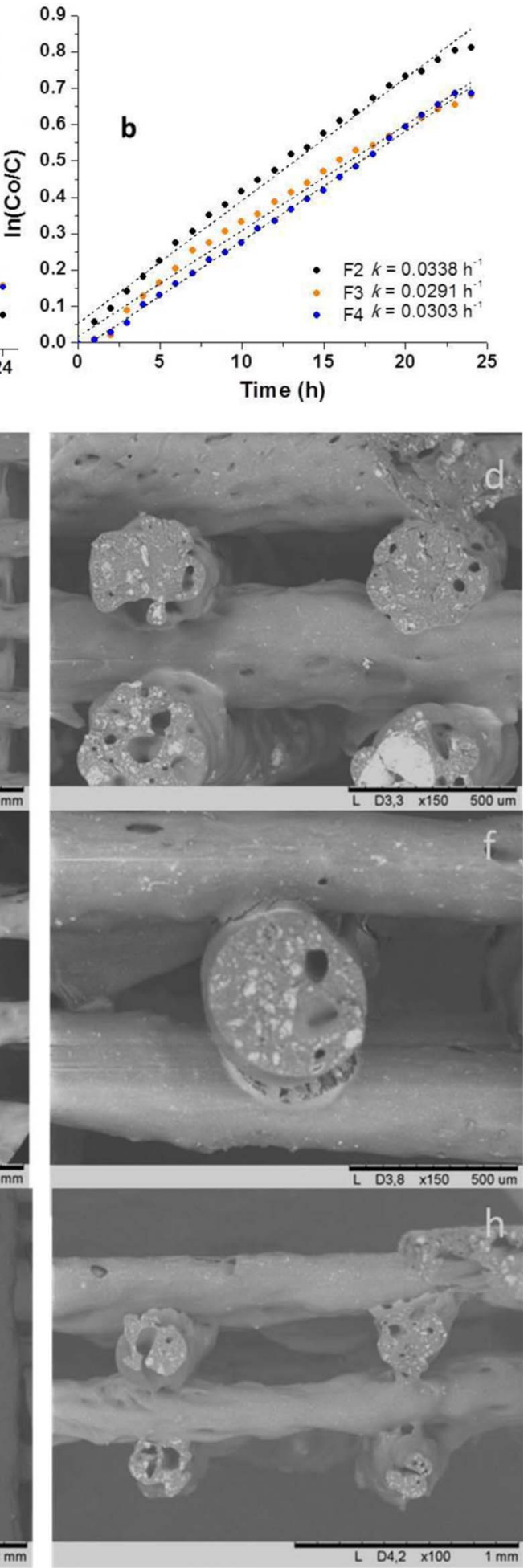

Figure 8. $(\mathrm{a}, \mathrm{b})$ Photodegradation of MO and kinetics of F2, F3 and F4 filters; (c, d) FESEM images of the F2 filter; (e, f) FESEM images of the F3 filter; (g, h) FESEM images of the F4 filter at different magnifications. layers) to the F7 sample, probably indicating a scale-up effect during the FDM deposition of the largest one. However, this microstructure composed of superficial voids and irregular surfaces perfectly matches the requirements needed to have a higher surface area in contact with the dye solution, thus justifying the good photocatalytic performance of the F7 filter. The kinetics of MO photodegradation obtained using these filters (Figure 7b) confirm that the F7 filter is the most performing one, however, looking at the relative curves, only the F6 filter follows a first-order kinetic model as well as F5 filter after the first $5 \mathrm{~h}$. F7 shows a different behavior that is ascribed to its unique microstructure, illustrated in Figures $7 \mathrm{e}, 7 \mathrm{~h}$ and $7 \mathrm{k}$.

Figures $8 \mathrm{a}$ and $8 \mathrm{~b}$ report the $\mathrm{MO}$ photodegradation of $15 \mathrm{TiO}_{2}$ filters designed with a diameter of $14 \mathrm{~mm}$ and 4 layers varying the infill from $30 \%$ to $20 \%$ (nozzle diameter of $0.3 \mathrm{~mm}$ ). They exhibit weights ranging $0.13-0.08 \mathrm{~g}$, corresponding to the filter F2, F3 and F4 in Table I respectively, which pictures are showed in the inset. In terms of photocatalytic degradation of the MO solution, higher infill \% values lead to the best performance, as in the F2 case (30\%). The initial dye concentration is reduced of about $80 \%$ during the test. In this case, 

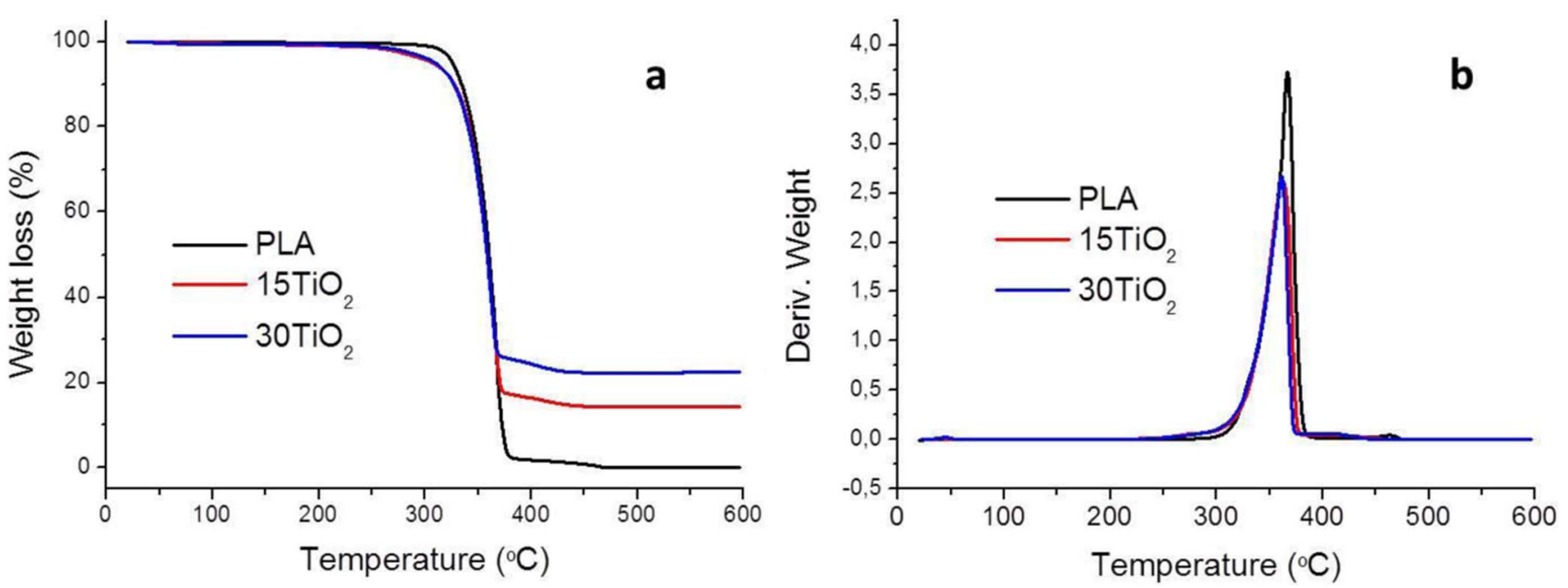

Figure 9. (a) Thermograms and (b) $\mathrm{TGA}$ derivate of $\mathrm{F} 3\left(15 \mathrm{TiO}_{2}\right)$ and $\mathrm{F} 7\left(30 \mathrm{TiO}_{2}\right)$ filters.

all the samples follow a first-order kinetic model with the F2 filter showing the highest $k$ value, equal to $0.0338 \mathrm{~h}^{-1}$. Keeping constant the rods wall roughness (Figures $8 \mathrm{c}, 8 \mathrm{e}$ and $8 \mathrm{~g}$ ) this trend mainly relies on the major amount of $\mathrm{TiO}_{2}$ present on the $\mathrm{F} 2$ filter, because of the higher mass, and thus the higher presence of photocatalytic sites. Figures $8 \mathrm{c}-8 \mathrm{~h}$ report the SEM images of the top view and the cross section of the filters F2 (c and d), F3 (e and f) and F4 ( $g$ and h) (in Table I). All the micrographs reported in Figures $8 \mathrm{c}-8 \mathrm{~h}$ show 3D structures composed of well-aligned rods that maintain their initial shape even without support of the underlying layer.

Finally, the stability of PLA/PEI-TiO $\mathrm{T}_{2}$ composites was measured by thermogravimetric test after the photodegradation test. Figure 9 illustrates the TGA (Figure 9a) and their respective derivative thermograms (DTGA) curves (Figure 9b) of pure PLA and both, $15 \mathrm{TiO}_{2}$ $(\mathrm{F} 3$, Figure $8 \mathrm{a})$ and $30 \mathrm{TiO}_{2}$ (F7, Figure 7a) filters, able to degrade the $70 \%$ and $100 \%$ of MO after $24 \mathrm{~h}$, respectively. Weight losses of the polymer and composites take place between 250 and $450^{\circ} \mathrm{C}$, with a maximum rate around $350^{\circ} \mathrm{C}$, evidencing that the composites degrade similarly to PLA. That means that the PLA matrix of the composites at least maintains its thermal properties after a double thermal process (extrusion and printing). The inorganic residue of the filters decomposition at $450^{\circ} \mathrm{C}$ is 15 and $25 \%$ of $15 \mathrm{TiO}_{2}$ and $30 \mathrm{TiO}_{2}$ composites, respectively, evidencing a slight loose of $\mathrm{TiO}_{2}$ content of the $30 \mathrm{TiO}_{2}$ filters during their double thermal processing, i.e. at the extrusion of the composites filaments and the FDM printing of filters.

The results of a detailed study of the filters after operation, in particular the initial degradation temperature $\left(T_{0}\right)$, the maximum degradation temperature $\left(T_{\max }\right)$ and the final degradation temperature $\left(T_{e}\right)$ data, are summarized in Table II. The maximum peaks in the DTGA curves represent the temperatures at maximum degradation rates $\left(\mathrm{T}_{\max }\right)$. Although the PLA is a biodegradable polymer its degradation properties are influenced by different factors such as polymer crystallinity, chemical bond, temperature or UV radiation. ${ }^{35}$ The results show that the composite thermal degradation happens at practically the same temperature as the pure PLA therefore no photodegradation of the polymer matrix is observed, induced by the $\mathrm{TiO}_{2}$ nanoparticles, since

Table II. Thermal degradation temperatures: initial degradation $\left(T_{0}\right)$, maximum degradation $\left(T_{\max }\right)$ and the end of degradation $\left(T_{e}\right)$ temperatures.

\begin{tabular}{cccc} 
& $\mathrm{T}_{0}\left({ }^{\circ} \mathrm{C}\right)$ & $\mathrm{T}_{\max }\left({ }^{\circ} \mathrm{C}\right)$ & $\mathrm{T}_{\mathrm{e}}\left({ }^{\circ} \mathrm{C}\right)$ \\
\hline $\mathrm{PLA}$ & 338 & 368 & 376 \\
$15 \mathrm{TiO}_{2}$ & 336 & 361 & 371 \\
$30 \mathrm{TiO}_{2}$ & 336 & 362 & 368
\end{tabular}

MO is more susceptible to oxidation. Moreover, contrary to what could be expected, the PLA matrix doesn't seem to be degraded after $24 \mathrm{~h}$ dipped in a MO aqueous solution. In this sense, the heat barrier role of $\mathrm{TiO}_{2}$ particles ${ }^{36}$ in these composites should be considered since the inorganic load addition could mask in the thermal tests a slightly PLA degradation by dissolution in the aqueous solvent during the photodegradation test. On the other hand, the improvement of mechanical properties could be expected due to the increase of the content on $\mathrm{TiO}_{2}$ in the feedstock. ${ }^{37}$ However a higher amount of inorganic particles in the polymeric matrix promotes printing irregularities such as the closed porosity and voids at the bars core as well as the formation of the wall-porosity. Although these last microstructural defects step up the photocatalytic activity of the filters they could compromise the mechanical properties improvement. Consequently, once the thermal degradation of the polymeric matrix has been avoided by substituting the thermal mixing of the PLA and $\mathrm{TiO}_{2}$ NPs by the proposed colloidal processing for the feedstock preparation, further research should be addressed to optimize the filters mechanical properties while maintaining their photocatalytic performance. In view of the obtained results, we can assume that it is possible to process scaffolds with an improved photocatalytic response retaining the composite structure at least during the photodegradation test, and hence keeping immobilized the photocatalyst.

\section{Conclusions}

Eco-friendly and biopolymer-based composites, able to photodegrade an organic molecule, were designed for 3D printing of photocatalytic filters. 3D scaffolds with an engineered microstructure and containing immobilized $\mathrm{TiO}_{2}$ NPs in PLA, were for the first time printed by fused filament fabrication or fused deposition modeling (FDM) with low-cost 3D printers. FDM allows the manufacture of customized $3 \mathrm{D}$ pieces, and in this work, the variation of the $3 \mathrm{D}$ scaffold geometry, by manipulating the printer parameters, leads to achieve the $100 \%$ degradation of $\mathrm{MO}$ for 70:30 (wt/wt) PLA:PEI-TiO 2 composites.

New polymer composites were produced by incorporating modified PEI-TiO ${ }_{2}$ NPs in a thermoplastic biopolymer such as PLA. PEI adsorption onto the nanopowders surface provides the needed stability to disperse NPs during the whole process, from the mixture of the PLA and $\mathrm{TiO}_{2}$ NPs in a THF suspension to the final homogenous distribution of $\mathrm{TiO}_{2}$ in the PLA matrix at the FDM scaffolds. Moreover, the surface modification of the $\mathrm{TiO}_{2}$ NPs allows increasing the inorganic loading of the polymer minimizing charge losses during the manufacturing of filaments and scaffolds by the extrusion and printing for the composites formulated with the highest $\mathrm{TiO}_{2}$ contents (70:30 (wt/wt) PLA:PEI-TiO ${ }_{2}$ ). The increase of the inorganic charge 
in the polymer as well as the scaling up parameters used produce to a valuable wall roughness/porosity in the scaffolds rods that propitiates the photocatalysis.

\section{Acknowledgments}

Authors acknowledge the support to the projects S2018/NMT4411 (Comunidad de Madrid) and MAT2015-70780-C4-1 (MINECO/FEDER). Z. Gonzalez acknowledges the Spanish Ministry of Economy and Competitiveness for the Postdoctoral Fellowship: IJCI-2016-28538. J. Yus acknowledges to the Comunidad de Madrid the support from the Youth Employment Initiative, CAMPD17_ICV_002. The authors thank ECERS for funding on Mobility Project JECS Trust Contract number: 2017294.

\section{ORCID}

B. Ferrari (D) https://orcid.org/0000-0003-3377-6844

\section{References}

1. A. Sobczyński and A. Dobosz, Polish J. Environ. Stud., 10, 195 (2001).

2. O. Legrini, E. Oliveros, and A. M. Braun, Chem. Rev., 93, 671 (1993).

3. A. Y. Shan, T. I. M. Ghazi, and S. A. Rashid, Appl. Catal. A Gen., 389, 1 (2010).

4. P. Esparza, M. E. Borges, L. Díaz, M. C. Alvarez-Galván, and J. L. G. Fierro, Appl. Catal. A Gen., 388, 7 (2010).

5. B. Zhu and L. Zou, J. Environ. Manage., 90, 3217 (2009).

6. L. Jin and B. Dai, Appl. Surf. Sci., 258, 3386 (2012).

7. E. Stathatos, D. Papoulis, C. A. Aggelopoulos, D. Panagiotaras, and A. Nikolopoulou, J. Hazard. Mater., 211-212, 68 (2012).

8. G. Li, S. Park, D. W. Kang, R. Krajmalnik-Brown, and B. E. Rittmann, Environ. Sci. Technol., 45, 8359 (2011).
9. L. Djafer, A. Ayral, and A. Ouagued, Sep. Purif. Technol., 75, 198 (2010).

10. C. P. Athanasekou et al., J. Memb. Sci., 392-393, 192 (2012).

11. G. E. Romanos et al., J. Hazard. Mater., 211-212, 304 (2012).

12. R. A. Damodar, S.-J. You, and H.-H. Chou, J. Hazard. Mater., 172, 1321 (2009).

13. L. Liu, Z. Liu, H. Bai, and D. D. Sun, Water Res., 46, 1101 (2012).

14. N. M. Bedford, M. Pelaez, C. Han, D. D. Dionysiou, and A. J. Steckl, J. Mater. Chem., 22, 12666 (2012).

15. D. L. Bourell, M. H. L., J. W. Barlow, J. J. Beaman, and C. R. Deckard, (1991).

16. K. Prashantha and F. Roger, J. Macromol. Sci. Part A Pure Appl. Chem., 54, 24 (2017).

17. M. R. Skorski, J. M. Esenther, Z. Ahmed, A. E. Miller, and M. R. Hartings, Sci. Technol. Adv. Mater., 17, 89 (2016).

18. L. E. Murr et al., Philos. Trans. R. Soc. A Math. Phys. Eng. Sci., 368, 1999 LP-2032 (2010).

19. S. J. Leigh, R. J. Bradley, C. P. Purssell, D. R. Billson, and D. A. Hutchins, PLoS One, 7, e49365 (2012).

20. W. Zhong, F. Li, Z. Zhang, L. Song, and Z. Li, Mater. Sci. Eng. A, 301, 125 (2001).

21. F. Ning, W. Cong, J. Qiu, J. Wei, and S. Wang, Compos. Part B Eng., 80, 369 (2015).

22. Y. Wu, D. Isakov, and P. S. Grant, Materials (Basel)., 10 (2017).

23. C. Shemelya et al., IEEE Sens. J., 15, 1280 (2015).

24. C. M. Shemelya et al., J. Electron. Mater, 44, 2598 (2015).

25. M. S. de, S. B. Monteiro, and M. I. B. Tavares, Adv. Nanoparticles, 07, 11 (2018).

26. A. Muñoz-Bonilla et al., Int. J. Mol. Sci., 14, 9249 (2013).

27. Z. Gonzalez et al., J. Eur. Ceram. Soc., 39, 366 (2019).

28. Y. Castro and A. Durán, J. Sol-Gel Sci. Technol., 78, 482 (2016).

29. J. A. Lewis, J. Am. Ceram. Soc., 83, 2341 (2004).

30. A. Ferrandez-Montero, A. Ferrari, B. Sanchez-Herencia, A. J. Gonzalez, Z. Gonzalez, F. J. Yus, J. Lieblich, and J. L. Gonzalez, ES1641.1356, Regist. Date 24/05/2018, 233.

31. P. Pan, B. Zhu, W. Kai, T. Dong, and Y. Inoue, J. Appl. Polym. Sci., 107, 54 (2008).

32. H. Salas-Papayanopolos, A. B. Morales-Cepeda, S. Sanchez, P. G. Lafleur, and I. Gomez, Polym. Bull., 74, 4799 (2017).

33. Y. Li, X. Li, J. Li, and J. Yin, Water Res., 40, 1119 (2006).

34. M. N. Rashed and A. A. El-Amin, 2, 73 (2007).

35. R. Auras, L. T. Lim, S. E. M. Selke, and H. Tsuji, Poly-lactic acid. Synthesis, Structures, Properties, and Applications, John Wiley \& Sons, Inc., New Jersey, (2010).

36. H. Zhang et al., RSC Adv., 5, 4639 (2015).

37. Y. Luo et al., J. App.Polym. Sci., 46509, 1 (2018). 